\title{
Calidad nutricional de un producto extruido fortificado con dos niveles de hierro proveniente de harina de sangre bovina
}

\section{Nutritional quality of an extruded product fortified with two levels of iron from bovine blood meal}

\author{
Ronny Galarza Martel', Yadira Cairo Arellano² \\ ${ }^{1}$ Universidad Nacional Mayor de San Marcos (UNMSM), Policlínico Los Olivos EsSalud. \\ ${ }^{2}$ Facultad de Medicina, Escuela Académico Profesional de Nutrición, UNMSM.
}

DOI: https://doi.org/10.33017/RevECIPeru2013.0009/

\section{Resumen}

La anemia por deficiencia de hierro continúa siendo uno de los principales problemas de salud pública, especialmente en los países en vías de desarrollo donde las dietas presentan bajo contenido de hierro hemínico. Los alimentos de origen animal como la sangre bovina son una fuente de alto contenido de hierro y de mayor biodisponibilidad por contener hierro hemínico y su deshidratación sería útil para la fortificación de productos extruidos. El objetivo de la investigación fue determinar la calidad nutricional de un producto extruido fortificado con dos niveles de hierro proveniente de harina de sangre bovina. Se realizó un estudio de tipo tecnológico experimental donde se obtuvo harina de sangre bovina a partir de un secado por atomización con ello se formuló dos niveles de fortificación de $10 \%$ y $15 \%$ además un producto sin fortificar $(0 \%)$ y para la obtención del producto extruido se utilizó un extrusor de un tornillo sinfín en un rango de temperatura entre $158{ }^{\circ} \mathrm{C}$ a $162{ }^{\circ} \mathrm{C}$ en la planta piloto de la Facultad de Industrias Alimentarias de la Universidad Nacional Agraria La Molina (UNALM). La calidad nutricional se determinó a partir del contenido de hierro, además se realizó el análisis proximal, físico-químico, microbiológico y en la prueba de aceptabilidad participaron 60 escolares de la Institución Educativa "Manuel González Prada N²015" los cuales fueron agrupados en 2 paneles: $\mathrm{N}^{\circ} 1$ : De 5 a 6 años de edad y el $\mathrm{N}^{\circ} 2$ : De 13 a 15 años de edad. Con respecto a los resultados los productos extruidos fortificados con $10 \%$ y $15 \%$ presentaron un elevado contenido de hierro $31,87 \mathrm{mg} / 100 \mathrm{~g}$ y $38,08 \mathrm{mg} / 100 \mathrm{~g}$ respectivamente a diferencia de un menor contenido de este en el producto extruido sin fortificar (0\%) que fue de $2,99 \mathrm{mg} / 100 \mathrm{~g}$. Según el análisis proximal el contenido proteínas fue mayor en los productos extruidos fortificados con $10 \%$ y $15 \%(12,47 \mathrm{~g} / 100 \mathrm{~g}$ y $13,80 \mathrm{~g} / 100 \mathrm{~g}$ respectivamente) que el producto sin fortificar $7,19 \mathrm{~g} / 100 \mathrm{~g}$. Mediante el análisis físico-químico se determinó el índice de expansión que fue mediana para el producto extruido sin fortificar $(0 \%)$ y fortificado con $10 \%$ y de expansión baja para el producto fortificado con 15\%. El análisis microbiológico demostró que los productos extruidos fueron aptos para el consumo humano. En la prueba de aceptabilidad al panel №1 se le presentó una escala hedónica de 3 puntos y se encontró que no hubo diferencia significativa entre las tres muestras de productos extruidos $(p<0,05)$ y al panel №2 se le presentó una escala hedónica de 5 puntos y se encontró que sí hubo diferencia significativa entre los productos extruidos de $0 \%$ y $15 \%$ de fortificación $(p<0,05)$. En conclusión el producto extruido fortificado con $10 \%$ de hierro proveniente de harina de sangre bovina presentó una adecuada calidad nutricional y fue más aceptable que el producto extruido fortificado con 15\%. Consumiendo $40 \mathrm{~g}$ de este producto se cubrirían las recomendaciones de $12,6 \mathrm{mg}$ de hierro por día en niños de 4 a 6 años de edad y se podría prevenir la anemia por deficiencia de hierro.

Descriptores: Harina de sangre bovina, producto extruido fortificado, calidad nutricional, hierro hemínico. 


\section{Abstract}

Iron deficiency anemia remains a major public health problem especially in developing countries where diets have low heme iron. Foods of animal origin such as bovine blood are a rich source of high iron content and most bioavailability by contain heme iron and its dehydration would be useful for fortification of extruded products. The objective of the research was to determine the nutritional quality of an extruded product fortified with two levels of iron from a bovine blood meal. A type of experimental technological study was realized. Bovine blood meal was obtained from a spray drying it was formulated with two levels of fortification of $10 \%$ and $15 \%$ also an unfortified product $(0 \%)$ and for obtaining the extruded product was used a screw extruder auger at a temperature range between $158{ }^{\circ} \mathrm{C}$ to $162{ }^{\circ} \mathrm{C}$ in the Pilot Plant Food Industry Faculty of the Universidad Nacional Agraria La Molina (UNALM). The nutritional quality was determined from the iron content. Further proximate analysis, physical-chemical, microbiological was performed and acceptability test participated 60 students of Manuel González Prada $N^{\circ} 2015$ School, they were grouped into two panels: $N^{\circ} 1: 5$ to 6 years old and $\mathrm{N}^{\circ} 2: 13$ to 15 years old. Respect to the result the extruded products fortified with $10 \%$ and $15 \%$ of iron from the bovine blood meal, had a high iron content $31,87 \mathrm{mg} / 100 \mathrm{~g}$ and $38,08 \mathrm{mg} / 100 \mathrm{~g}$ respectively as opposed to a lower iron content in the extrudate without fortify (0\%) which was 2,99 $\mathrm{mg} / 100 \mathrm{~g}$. According to the proximate analysis was higher protein content extruded products fortified with $10 \%$ and $15 \%$ $(12.47 \mathrm{~g} / 100 \mathrm{~g}$ and $13.80 \mathrm{~g} / 100 \mathrm{~g}$, respectively) than $7.19 \mathrm{~g} / 100 \mathrm{~g}$ unfortified product. Through the physicochemical analysis the rate of expansion was determined. It was median for unfortified extruded product $(0 \%)$ and fortified with $10 \%$ and low expansion for product fortified with $15 \%$. Microbiological analysis demonstrated that the extruded products were fit for human consumption. In the acceptability test to panel №1 was presented a 3-point hedonic scale and there was no significant difference between the three samples of extruded $(p<0,05)$ and the panel №2 was presented a 5-point hedonic scale and there were significant differences between samples of extruded products with level of fortification of $0 \%$ and $15 \%(p<0,05)$. In conclusion, the extruded product fortified with $10 \%$ of iron from bovine blood meal showed an adequate nutritional quality and was more acceptable than the extruded product fortified with $15 \%$. Consuming $40 \mathrm{~g}$ of this product would meet the recommendations of $12,6 \mathrm{mg}$ of iron per day in children 4-6 years old and it could prevent iron-deficiency anemia.

Keywords: Bovine blood meal, extruded product fortified, nutritional quality, heme iron.

\section{Introducción}

La deficiencia de hierro continúa siendo uno de los principales problemas de salud pública asociados a la nutrición, especialmente en los países en vías de desarrollo donde las dietas tienen bajo contenido de hierro hemínico, siendo éste el principal factor que influye sobre su biodisponibilidad [1].

Las fuentes de proteínas tales como: la carne, el pescado y el pollo tienen un efecto positivo en la biodisponibilidad del hierro no hemínico llamado "factor cárnico" y se relaciona específicamente con la proteína de origen muscular y no con la proteína de origen animal en general por lo que el huevo y la leche quedan excluidos [2].

La población en mayor riesgo de presentar deficiencias de hierro son los lactantes, niños, adolescentes, mujeres en edad fértil y gestantes [3].

La desnutrición crónica y la anemia por deficiencia de hierro son los principales problemas nutricionales a nivel mundial que afecta a los niños en edad preescolar [3].
La prevalencia de anemia en el Perú fue de $34,9 \%$ en niños menores de 5 años según el Sistema de Información del Estado Nutricional (SIEN, CENAN 2012) resultados que continúan elevados. [4]

La dieta que reciben la mayoría de los niños no logra cubrir sus requerimientos de hierro, lo cual hace que aumente la prevalencia de anemia en nuestro país, perjudicando el crecimiento y desarrollo de los niños, además poniendo en riesgo su salud en general.

Teniendo conocimiento que los alimentos de origen animal como las carnes rojas, vísceras y la sangre son fuentes de alto contenido de hierro y de mayor biodisponibilidad por contener hierro hem [5], la sangre animal sería útil en sus diferentes formas de consumo, que señalan una alternativa para combatir la anemia, que en nuestro medio sólo se consume a través de la llamada sangrecita.

La sangre bovina por su contenido de proteínas (18\%) y su buena fuente de aminoácidos esenciales 
como la leucina, lisina y triptófano además de ser una fuente de hierro hemínico sería útil para la fortificación de alimentos. [5]

La deshidratación por secado por atomización tiene mayor preferencia debido a su mayor rendimiento, homogeneidad en el producto, además por las características del alimento que se altera mínimamente sin ocasionar daños en su composición [6]. De esta manera es un proceso fundamental para la obtención de harina de sangre bovina.

La tecnología en la extrusión de alimentos tiene muchas ventajas por su alta productividad al procesar gran cantidad de alimentos de manera continua en un corto tiempo utilizando un equipo sofisticado, por su ahorro de energía y mano de obra en su sistema de cocción a un bajo costo, por su calidad y capacidad de producir nuevos alimentos a partir de diferentes formas, colores y texturas, lo que le permite una amplia aceptabilidad, además por presentar una prologada vida útil y de consumo directo [7]. En nuestro país los productos extruidos llegan bajo la forma de snacks, corn flakes e incluso los chizitos que se consumen y tienen alta preferencia en nuestra población infantil [7].

La extrusión es un proceso en que una mezcla de alimentos es forzada a fluir, en virtud a parámetros adecuados, de las condiciones de mezcla, humedad, temperatura y corte a través de un troquel que está diseñado para dar formar al producto final $[6,8]$.

Por lo mencionado anteriormente la presente investigación tuvo como objetivo determinar la calidad nutricional de un producto extruido fortificado con dos niveles de hierro proveniente de harina de sangre bovina, cuyo contenido de hierro pudiera tomarse en cuenta para prevenir y disminuir la anemia por deficiencia de hierro que presenta nuestra población infantil.

\section{Metodología}

Se desarrolló una investigación de tipo tecnológico, en el cual se realizó dos niveles de fortificación $(10 \%$ y $15 \%)$ con harina de sangre bovina para obtener un producto extruido los cuales fueron hechos en la Planta piloto de la Facultad de Industrias Alimentarias de la UNALM. Para determinar la calidad nutricional de los productos extruidos se determinó el contenido de hierro, el análisis proximal, físico-químico y microbiológico que se analizaron en el Centro de Control Analítico de la
Facultad de Farmacia y Bioquímica de la UNMSM, además se realizó la evaluación sensorial de los productos en la Institución Educativa "Manuel González Prada N²015" para cada nivel de fortificación incluyendo el producto sin fortificar.

Los Materiales y equipos del proceso fueron:

- Secador por atomización: Secador Spray Dried, Modelo A81, Marca Aromas del Perú S.A.

- Anticoagulante de Citrato de sodio $3 \mathrm{~g} / \mathrm{L}$ de sangre bovina.

- Extrusor de alimentos: De un tornillo sinfín simple con sistema de alimentación por gravedad. Facultad de Industrias Alimentarias, UNALM.

- Analizador de Humedad: Modelo MJ33 MettlerToledo.

- Balanza digital de alimentos marca Soehnle con una precisión $0,1 \mathrm{~g}$.

- Molino manual con discos para granos.

- Recipientes de vidrio y baldes herméticos.

- Materiales de empaque: bolsas de polietileno hermético, tápers de plásticos y etiquetas.

\subsection{Obtención de la harina de sangre bovina}

La recolección de la sangre bovina provino de aquellos animales que fueron sometidos a inspección veterinaria antes de ser sacrificados para que sean aptos para el consumo humano. Para impedir la coagulación de la sangre se utilizó citrato de sodio en la proporción de $3 \mathrm{~g}$ por litro de sangre mezclándola por 2 minutos para mantenerla en estado líquido.

Posteriormente se trasladó en vasijas la sangre de bovino para su deshidratación a través de un secado por atomización (Secador Spray Dried, Modelo A81), con los siguientes parámetros de secado: temperatura de entrada a $165^{\circ} \mathrm{C}$ y temperatura de salida a $75 \stackrel{\circ}{\circ}$ a una presión de 1 atmósfera por un tiempo de 40 minutos, de esta manera se obtuvo la harina de sangre bovina, además este tratamiento térmico aseguró la estabilidad microbiológica haciéndola apta para el consumo [6]. Posteriormente se realizó el análisis proximal y del contenido de hierro de la harina de sangre bovina en los laboratorios de Certificaciones del Perú S.A. (CERPER).

\subsection{Niveles de fortificación}

El producto extruido se formuló proyectándose a situaciones adversas que limitan la biodisponibilidad 
del hierro para cubrir las recomendaciones de niños de 4 a 6 años de edad según la Organización Mundial de la Salud (OMS) 2004 [8]. Se realizaron pruebas piloto para la elaboración del producto extruido, a partir de éstas se eligió los dos niveles de fortificación con hierro proveniente de harina de sangre bovina de $10 \%$ y $15 \%$, además se elaboró un producto extruido sin fortificar como muestra de comparación.

\subsection{Elaboración del producto extruido}

La elaboración del producto extruido se realizó en la Planta Piloto de la Facultad de Industrias Alimentarias de la Universidad Nacional Agraria La Molina (UNALM). Se recepcionó la materia prima herméticamente sellada además de los principales insumos: granos de trigo, arroz partido, el grits de maíz, azúcar rubia y esencia de chocolate. Luego se seleccionaron los insumos para su molienda, tamizado y mezclado acondicionándolo a una humedad de $11 \%$. Se utilizó un extrusor de alimentos de un tornillo sinfín con un sistema de alimentación por gravedad y con un troquel de 4 orificios. La mezcla de ingredientes se ingresó por la tolva y se comenzó a procesar a una temperatura entre los $158{ }^{\circ} \mathrm{C}$ a $162 \stackrel{\circ}{\circ} \mathrm{C}$ con una velocidad del tornillo de 254,5 rpm. Posteriormente se dejó enfriar $y$ se empacaron en bolsas de polietileno debidamente selladas.

\subsection{Evaluación de la calidad nutricional}

Se evaluó principalmente a través del contenido total de hierro y se estimó su biodisponibilidad de acuerdo al contenido de hierro hem y no hem.

\subsubsection{Análisis del contenido de hierro:}

Se realizó en las tres muestras de productos extruidos de acuerdo al método de Espectrofotometría de absorción atómica de la Association of Official Analytical Chemist (AOAC) en el Centro de Control Analítico de la Facultad de Farmacia y Bioquímica de la Universidad Nacional Mayor de San Marcos (UNMSM).

Se determinó el hierro biodisponible utilizando el siguiente algoritmo:

Hierro biodisponible $=($ Hierro hemínico $\times 0.25)+$ (Hierro no hemínico $x$ Factor biodisponibilidad $x$ Factor té o café)
Donde: El factor inhibidor (té/café) se utilizaron: "1" si la persona no toma dichas bebidas y "0,40" si se consume al menos $600 \mathrm{ml}$ diarios [2].

\subsubsection{Análisis Proximal}

Se determinó el contenido de proteínas, grasas, carbohidratos, fibra y cenizas el análisis proximal de acuerdo al método de la AOAC.

\subsubsection{Análisis físico-químicos}

Se determinó a través del índice de absorción de agua, índice de solubilidad en agua e índice de expansión [9].

\subsubsection{Análisis microbiológico}

Se realizó de acuerdo a la Norma técnica de Salud $N^{\circ} 071$ "Norma sanitaria que establece los criterios microbiológicos de calidad sanitaria e inocuidad para los alimentos y bebidas de consumo humano" de la Dirección General de Salud Ambiental del Ministerio de Salud, que comprende la evaluación de agentes microbianos como: Aerobios mesófilos, Mohos, Levaduras, Coliformes, Bacillus cereus y Salmonella sp. [10]

\subsubsection{Prueba de Aceptabilidad}

Se analizaron los atributos de sabor, olor, textura (crocantés) y aspecto de los productos extruidos además la aceptabilidad según el nivel de fortificación proveniente de harina de sangre bovina el cual se utilizó la prueba hedónica para medir cuanto agrada o desagrada el producto empleando dos formas de escalas categorizadas, de 3 puntos que van desde: Me disgusta, ni me gusta ni me disgusta, me gusta y de 5 puntos que van desde: Me disgusta mucho, me disgusta, ni me gusta ni me disgusta, me gusta, me gusta mucho [7,11]. Participaron como panelistas 60 escolares de la Institución Educativa "Manuel González Prada N²015" y se les agrupó en 2 paneles: №1: De 5 a 6 años de edad y el №2: De 13 a 15 años de edad.

\subsection{Análisis Estadístico}

Con los datos obtenidos en la prueba organoléptica y de aceptabilidad de los productos extruidos según el nivel de fortificación, se digitó y procesó en el programa SPSS v.18 aplicando la prueba estadística de análisis de varianza para un nivel de significación de 0,05 , además se utilizó la prueba de Duncan como prueba post hoc. 


\section{Resultados y discusión}

La harina de sangre bovina obtenida del secado por atomización fue de $4,7 \mathrm{Kg}$ con una humedad del $3,67 \%$ a partir de la recolección de 27 litros de sangre de bovino, es decir el rendimiento fue de 174 $\mathrm{g}$ de harina de sangre bovina por litro de sangre. En la Tabla 1 se muestra la composición proximal de la harina de sangre bovina

Tabla 1. Composición proximal y contenido de hierro de la harina de sangre bovina en $100 \mathrm{~g}$.

\begin{tabular}{|c|c|}
\hline Análisis & Resultados \\
\hline Humedad $(\mathrm{g} / 100 \mathrm{~g})$ & 3,67 \\
\hline Proteína $(\mathrm{g} / 100 \mathrm{~g})$ & 91,8 \\
\hline Grasa $(\mathrm{g} / 100 \mathrm{~g})$ & 1,09 \\
\hline Ceniza $(\mathrm{g} / 100 \mathrm{~g})$ & 3,44 \\
\hline Carbohidratos $(\mathrm{g} / 100 \mathrm{~g})$ & 0,0 \\
\hline Hierro $(\mathrm{mg} / 100 \mathrm{~g})$ & 210,39 \\
\hline Energía total $(\mathrm{Kcal} / 100 \mathrm{~g})$ & 377,01 \\
\hline
\end{tabular}

Según Lucas O. (2005) quien también utilizó sangre bovina secada por atomización, obtuvo $146 \mathrm{~g}$ de harina por litro de sangre recolectada, cuyo contenido de proteínas fue de $83,71 \mathrm{~g} / 100 \mathrm{~g}$ y de hierro $385,2 \mathrm{mg} / 100 \mathrm{~g}[5]$ resultados diferentes a esta investigación que obtuvo un mayor rendimiento de harina de sangre bovina y de proteínas

Con respecto al contenido de hierro de los productos extruidos, éste se incrementó conforme aumentaba el nivel de fortificación. (Tabla 2)

Tabla 2: Contenido de hierro de los productos extruidos según el nivel de fortificación en $100 \mathrm{~g}$.

\begin{tabular}{|c|c|c|c|}
\hline & \multicolumn{3}{|c|}{$\begin{array}{c}\text { Nivel de fortificación } \\
\text { del producto extruido }\end{array}$} \\
\hline & $0 \%$ & $10 \%$ & $15 \%$ \\
\hline $\begin{array}{c}\text { Contenido de Hierro } \\
\text { (mg/100g) }\end{array}$ & 2,99 & 31,87 & 38,08 \\
\hline
\end{tabular}

Lucas O. realizó dos tipos de fortificaciones de galletas con harina de sangre bovina: $5 \%$ y $8 \%$ y un grupo control, que presentaron $20,96 \mathrm{mg} / 100 \mathrm{~g}$ $24,04 \mathrm{mg} / 100 \mathrm{~g}$ y $8,32 \mathrm{mg} / 100 \mathrm{~g}$ respectivamente [5], de los cuales fue mayor el contenido de hierro del producto extruido fortificados.

En la Tabla 3 se observa que los productos extruidos con $10 \%$ y $15 \%$ de fortificación tuvieron mayor contenido de hierro estimado proveniente de la harina de sangre bovina que el hierro proveniente de los demás insumos.

Tabla 3. Contenido de hierro total y estimación de hierro proveniente de la harina de sangre bovina $y$ de los demás insumos según el nivel de fortificación del producto extruido.

\begin{tabular}{|cc|c|c|}
\hline & \multicolumn{3}{c|}{$\begin{array}{c}\text { Nivel de fortificación } \\
\text { del producto extruido }\end{array}$} \\
\hline $\begin{array}{c}\text { Prueba } \\
\text { Hierro proveniente de harina de } \\
\text { sangre bovina }(\mathrm{mg} / 100 \mathrm{~g})^{*}\end{array}$ & 0,0 & $\mathbf{1 0} \%$ & $\mathbf{1 5} \%$ \\
\hline $\begin{array}{c}\text { Hierro proveniente de los demás } \\
\text { insumos }(\mathrm{mg} / 100 \mathrm{~g})^{*}\end{array}$ & 2,99 & 2,69 & 35,54 \\
\hline Hierro total $(\mathrm{mg} / 100 \mathrm{~g})^{* *}$ & 2,99 & 31,87 & 38,08 \\
\hline
\end{tabular}

* Estimación del contenido de hierro a partir del hierro total.

** Cantidad de hierro obtenido en laboratorio.

Con respecto al contenido de hierro biodisponible estimado de los productos extruidos se proyectó para situaciones desfavorables se consideró un factor inhibidor de 0,40 y un factor de biodisponibilidad de 5\% [2] en donde los productos extruidos fortificados con $10 \%$ y $15 \%$ de harina de sangre bovina aportaron mayor cantidad de hierro biodisponible estimado que el producto extruido sin fortificar (0\%). (Tabla 4)

Tabla 4: Estimación del contenido de hierro biodisponible de los productos extruidos según el factor de biodisponibilidad y el factor inhibidor.

\begin{tabular}{|c|c|c|c|c|c|c|}
\hline \multirow{4}{*}{ Prueba } & \multicolumn{6}{|c|}{$\begin{array}{l}\text { Nivel de fortificación del producto } \\
\text { extruido }\end{array}$} \\
\hline & \multicolumn{2}{|c|}{$0 \%$} & \multicolumn{2}{|c|}{$10 \%$} & \multicolumn{2}{|c|}{$15 \%$} \\
\hline & \multicolumn{2}{|c|}{$\begin{array}{l}\text { Factor de } \\
\text { Biodisp. }\end{array}$} & \multicolumn{2}{|c|}{$\begin{array}{l}\text { Factor de } \\
\text { biodisp. }\end{array}$} & \multicolumn{2}{|c|}{$\begin{array}{l}\text { Factor de } \\
\text { biodisp. }\end{array}$} \\
\hline & $5 \%$ & $10 \%$ & $5 \%$ & $10 \%$ & $5 \%$ & $10 \%$ \\
\hline $\begin{array}{c}\text { Factor inhibidor: } \\
0,40 \\
\text { Hierro } \\
\text { biodisponible } \\
(\mathrm{mg} / 100 \mathrm{~g})\end{array}$ & 0,05 & 0,11 & 3,32 & 3,72 & 4,03 & 4,50 \\
\hline $\begin{array}{c}\text { Factor inhibidor: } 1 \\
\text { Hierro } \\
\text { biodisponible } \\
(\mathrm{mg} / 100 \mathrm{~g})\end{array}$ & 0,14 & 0,29 & 3,92 & 4,93 & 4,74 & 5,94 \\
\hline
\end{tabular}

Con respecto a la composición proximal de los productos extruidos el contenido proteínas fue mayor en los productos extruidos fortificados esto se debe 
al alto contenido proteico que presenta la harina de sangre bovina, a la vez el aporte energético varía ligeramente en las tres muestras de productos extruidos tal como se observa en la Tabla 5.

Tabla 5: Composición proximal de los productos extruidos según nivel de fortificación en $100 \mathrm{~g}$.

\begin{tabular}{|c|c|c|c|}
\hline \multirow{2}{*}{ Análisis } & \multicolumn{3}{c}{$\begin{array}{c}\text { Nivel de fortificación del } \\
\text { producto extruido }\end{array}$} \\
\cline { 2 - 4 } & $\mathbf{0 \%}$ & $\mathbf{1 0} \%$ & $\mathbf{1 5} \%$ \\
\hline Humedad $(\mathrm{g} / 100 \mathrm{~g})$ & 6,63 & 5,57 & 5,00 \\
\hline Proteína $(\mathrm{g} / 100 \mathrm{~g})$ & 7,19 & 12,47 & 13,80 \\
\hline Grasa $(\mathrm{g} / 100 \mathrm{~g})$ & 4,39 & 3,20 & 4,00 \\
\hline Ceniza $(\mathrm{g} / 100 \mathrm{~g})$ & 2,85 & 2,11 & 2,36 \\
\hline Carbohidratos $(\mathrm{g} / 100 \mathrm{~g})$ & 74,29 & 70,55 & 66,62 \\
\hline Fibra $(\mathrm{g} / 100 \mathrm{~g})$ & 4,65 & 6,10 & 8,22 \\
\hline $\begin{array}{c}\text { Energía total } \\
(\text { Kcal/100 g) }\end{array}$ & 365,43 & 360,88 & 357,68 \\
\hline
\end{tabular}

En los análisis físico-químico, el índice de expansión disminuyó conforme el nivel de fortificación con harina de sangre bovina aumentaba, sin embargo, los productos extruidos sin fortificar (0\%) y fortificado con $10 \%$ tuvieron una mediana expansión a diferencia del producto extruido fortificado con $15 \%$ que obtuvo una baja expansión. (Tabla 6)

Tabla 6. Índice de absorción de agua, índice de solubilidad en agua e índice de expansión de los productos extruidos según el nivel de fortificación.

\begin{tabular}{|c|c|c|c|}
\multirow{2}{*}{ Análisis } & \multicolumn{3}{|c|}{$\begin{array}{c}\text { Nivel de fortificación del } \\
\text { producto extruido }\end{array}$} \\
\cline { 2 - 4 } & $\mathbf{0} \%$ & $\mathbf{1 0} \%$ & $\mathbf{1 5} \%$ \\
\hline $\begin{array}{c}\text { Índice de absorción } \\
\text { de agua }\end{array}$ & 3,8 & 3,6 & 3,7 \\
\hline $\begin{array}{c}\text { Índice de } \\
\text { solubilidad en agua }\end{array}$ & 0,04 & 0,16 & 0,12 \\
\hline \multirow{2}{*}{$\begin{array}{c}\text { Índice de } \\
\text { expansión* }\end{array}$} & 1,93 & 1,61 & 1,38 \\
\cline { 2 - 4 } & Mediana & Mediana & Baja \\
\hline
\end{tabular}

${ }^{*}$ Baja: $<1,5 \quad$ Media: 1,6 - $2 \quad$ Alta: $>2$

Kameko J. (2005) obtuvo un producto extruido a base de olluco-quinua que su índice de solubilidad en agua fue de 0,503, sin embargo, en el presente trabajo se encontró en los productos fortificados con $10 \%$ y $15 \%$ un menor índice de solubilidad en agua esta diferencia puede deberse a la elevada velocidad del tornillo y a la generación de fricción molecular del cilindro del extrusor produciéndose dextrinización de almidones, de tal manera que se liberarían compuestos de bajo peso molecular aumentando su solubilidad; de esta manera existe una relación inversa, a medida que aumente el índice de absorción de agua disminuye el índice de solubilidad en agua. [7]

Los resultados del análisis microbiológico de los productos extruidos sin fortificar $(0 \%)$ y fortificados con $10 \%$ y $15 \%$ de harina de sangre bovina se presentan en el Tabla 7, los cuales indican que los productos extruidos fueron aptos para el consumo humano, de acuerdo con los criterios microbiológicos establecidos por la Norma Técnica de Salud № 071.

Tabla 7. Análisis microbiológico de los productos extruidos según el nivel de fortificación con harina de sangre bovina.

\begin{tabular}{|c|c|c|c|c|c|}
\hline \multirow{2}{*}{ Pruebas } & \multicolumn{3}{|c|}{$\begin{array}{l}\text { Nivel de fortificación del } \\
\text { producto extruido }\end{array}$} & \multicolumn{2}{|c|}{ Límite por g. } \\
\hline & $0 \%$ & $10 \%$ & $15 \%$ & Mínimo & Máximo \\
\hline $\begin{array}{l}\text { Aeróbios } \\
\text { mesófilos } \\
\text { (ufc/g) }\end{array}$ & $<10$ & 35 & 35 & $10^{4}$ & $10^{5}$ \\
\hline $\begin{array}{l}\text { Mohos y } \\
\text { levaduras } \\
\text { (ufc/g) }\end{array}$ & 155 & 15 & 20 & $10^{2}$ & $10^{3}$ \\
\hline $\begin{array}{l}\text { Coliformes } \\
\text { (NMP/g) }\end{array}$ & 15 & 4 & 4 & 10 & $10^{2}$ \\
\hline $\begin{array}{l}\text { Bacillus } \\
\text { cereus } \\
\text { (en } 10 \mathrm{~g} \text { ) }\end{array}$ & Ausente & Ausente & Ausente & $10^{2}$ & $10^{4}$ \\
\hline $\begin{array}{c}\text { Salmonella } \\
\text { sp. } \\
\text { (en } 25 \mathrm{~g} \text { ) }\end{array}$ & Ausente & Ausente & Ausente & Ausente & Ausente \\
\hline
\end{tabular}

ufc: Unidad formadora de colonias. NMP: Número más probable.

Según el promedio de puntajes de la prueba de aceptabilidad por parte de los alumnos de 5 a 6 años de edad se obtuvo que no hay diferencia significativa (valor $p=0,426$ ) entre las tres muestras de productos extruidos $(0 \%, 10 \%, 15 \%)$ a un nivel de significancia de $p<0,05$. En dicha prueba de aceptabilidad se obtuvo un promedio por encima de los 2 puntos lo que nos indica que se encuentra más próximo a la escala "me gusta". (Figura 1)

Los resultados de la prueba organoléptica en los alumnos de 13 a 15 años de edad del nivel secundaria de la I.E. No 2015 "Manuel González Prada" (panel $\mathrm{N}^{\circ} 2$ ), mostraron que sí hay diferencia significativa entre los promedios de puntajes de sabor y olor en las muestras de productos extruidos con nivel de fortificación $0 \%$ y $15 \%$ a un nivel de significancia de $p<0,05$. En cambio, los promedios de los puntajes de crocantés y aspecto en las tres muestras de productos extruidos (0\%, $10 \%$ y $15 \%)$ 
no hubo diferencias significativas a un nivel de $\mathrm{p}<0,05$, tal como se muestra en la Tabla 8.

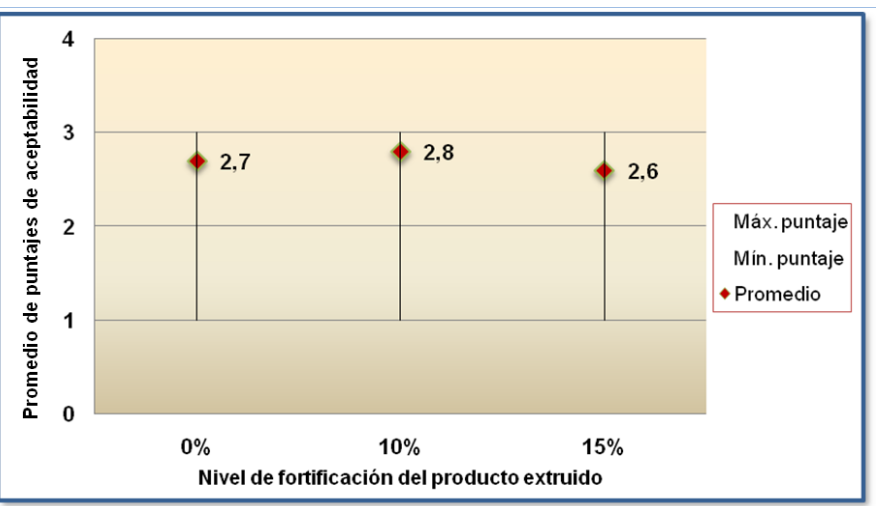

Figura 1. Promedio de puntajes de la prueba de aceptabilidad según el nivel de fortificación del producto extruido en alumnos de 5 a 6 años de edad del nivel primaria de la I.E. $N^{\circ} 2015$ "Manuel González Prada", 2010. ( $n=30)$

Tabla 8. Promedio y desviación estándar de la prueba organoléptica según el nivel de fortificación del producto extruido en alumnos de 13 a 15 años de edad del nivel secundaria de la l.E. № 2015 "Manuel González Prada", 2010. (n=30)

\begin{tabular}{|c|c|c|c|c|}
\hline \multirow{2}{*}{$\begin{array}{c}\text { Características } \\
\text { organolépticas }\end{array}$} & $\mathbf{0} \%$ & $\mathbf{1 0} \%$ & $\mathbf{1 5} \%$ & Valor \\
\hline Sabor & $4,60 \pm 0,62$ & $4,27 \pm 0,74$ & $4,0 \pm 0,64$ & $0,004^{*}$ \\
\hline Olor & $4,60 \pm 0,50$ & $4,30 \pm 0,65$ & $4,0 \pm 0,70$ & $0,002^{*}$ \\
\hline Crocantés & $4,33 \pm 0,71$ & $4,17 \pm 0,83$ & $3,97 \pm 0,77$ & 0,189 \\
\hline Aspecto & $4,23 \pm 0,68$ & $4,03 \pm 0,62$ & $3,97 \pm 0,89$ & 0,350 \\
\hline
\end{tabular}

* Existe diferencia significativa (Prueba de Duncan)

Según el promedio de puntajes de la prueba de aceptabilidad por parte de los alumnos de 13 a 15 años de edad se obtuvo que sí hay diferencia significativa (valor $p=0,004$ ) entre las muestras de los productos extruidos con nivel de fortificación de $0 \%$ y $15 \%$ a un nivel de significancia de $p<0,05$, pero no hay diferencia significativa entre las muestras $0 \%$ y $10 \%$. En dicha prueba de aceptabilidad se obtuvo un promedio por encima de los 4 puntos para los productos extruidos sin fortificar $(0 \%)$ y fortificado con $10 \%$, indicando que tiende aproximarse a la escala "me gusta mucho". (Figura 2)

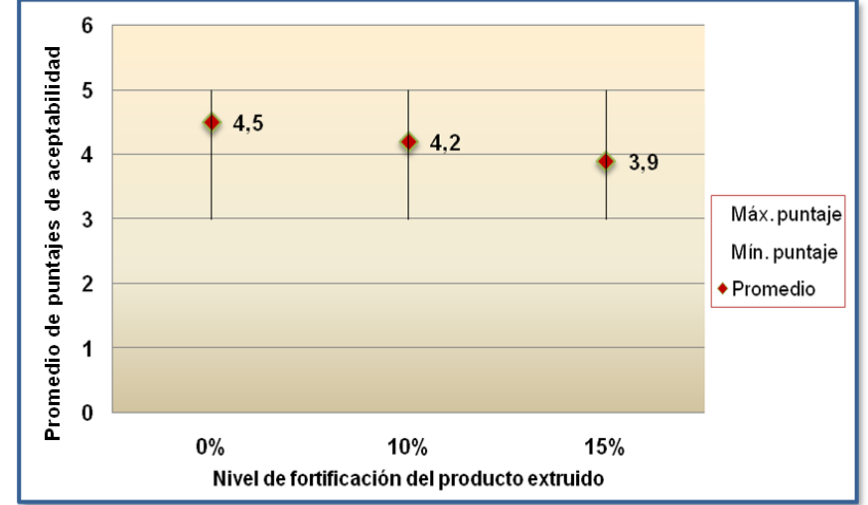

Figura 2. Promedio de puntajes de la prueba de aceptabilidad según el nivel de fortificación del producto extruido en alumnos de 13 a 15 años de edad del nivel secundaria de la I.E. N 2015 "Manuel González Prada", 2010. $(n=30)$

Con los resultados encontrados respecto al contenido de hierro de los productos extruidos se procedió a empacarlos en cantidades de $40 \mathrm{~g}$ de producto extruido fortificado con $10 \%$ y $33 \mathrm{~g}$ de producto extruido fortificado con $15 \%$ que representaron las cantidades que debería ser consumido para cubrir las recomendaciones de hierro en situaciones adversas que limitan la biodisponibilidad para niños de 4 a 6 años de edad, mientras estas mismas recomendaciones para el producto extruido sin fortificar $(0 \%)$ se requirió empaques con $421 \mathrm{~g}$.

\section{Conclusiones}

- Los dos niveles de fortificación con hierro proveniente de harina de sangre bovina en los productos extruidos fueron $10 \%$ y $15 \%$.

- Los productos extruidos fortificados con $10 \%$ y $15 \%$ presentaron mayor contenido de hierro y de proteínas que el producto extruido sin fortificar $(0 \%)$.

- Los productos extruidos fortificados con $10 \%$ y $15 \%$ cumplieron con los criterios microbiológicos establecidos por la Norma Técnica de Salud.

- El producto extruido fortificado con 10\% tuvo mayor aceptabilidad que el fortificado con $15 \%$ en los dos grupos de panelistas.

- El producto extruido fortificado con $10 \%$ de hierro proveniente de harina de sangre bovina presentó una adecuada calidad nutricional y fue más aceptable que el producto extruido fortificado con $15 \%$. 


\section{Recomendaciones}

El producto extruido con $10 \%$ de fortificación con harina de sangre bovina podría prevenir y resolver los problemas de anemia por deficiencia de hierro en niños de 4 a 6 años de edad consumiendo $40 \mathrm{~g}$ de este producto que cubrirían las recomendaciones de la OMS de 12,6 mg de hierro por día.

\section{Agradecimientos}

Agradecemos al Dr. Percy Lezama Vigo por su generosidad en la factibilidad de la materia prima. A la Dra. Teresa Arbaiza por sus sugerencias, al Ing. Oscar Crisóstomo G. de la UNALM por su amabilidad, conocimientos y gran ayuda en el proceso de extrusión. A los docentes de la Facultad de Medicina de la UNMSM.

\section{Referencias}

[1] D. Gaitán, M. Olivares, M. Arredondo, F. Pizarro. Biodisponibilidad de hierro en humanos. Rev. Chil. Nutr. 33(2006)142-148.

[2] R. González. Biodisponibilidad del hierro. Rev. Costarric. Salud Pública 14 (2005)16.

[3] N. Baiocchi. Anemia por deficiencia de hierro. Rev. Per. Pediatr. 59 (2006)20-27.

[4] Centro Nacional de Alimentación y Nutrición. Informe Gerencial Nacional SIEN, Periodo anual 2012. (Ministerio de Salud, 2012).

[5] O. Lucas. Evaluación nutricional de galletas fortificadas con sangre entera de bovino secada por atomización. Tesis para optar el grado de Magíster en Ciencias de los Alimentos. (Facultad de Farmacia y Bioquímica, UNMSM, Lima-Perú, 2005)

[6] P. Fellows. Tecnología del procesado de los alimentos: Principios y prácticas. (Zaragoza, España: Acribia, S. A, 1994), pp. 273-284.
[7] J. Kameko. Determinación de los parámetros de extrusión en un extrusor de bajo costo para la obtención de una mezcla base para desayuno a partir de oca (Oxalis tuberosa Mol.), olluco (Ullucus tuberosum Loz.) y quinua (Chenopodium quinoa Willd). Tesis para optar el título de Ingeniero en Industrias Alimentarias. (Facultad de Industrias Alimentarias, Universidad Nacional Agraria La Molina, 2005).

[8] World Health Organization. Food and Agricultural Organization of the United Nations. Vitamin and mineral requirements in human nutrition. 2da ed. (World Health Organization, 2004).

[9] J. Aro. Elaboración de una mezcla alimenticia a base de quinua (Chenopodium quinua Willd), Cañihua (Chenopodium pollidi Caulli), Cebada (Hordum vulgare L.), Maíz (Zea mayz L.), Haba (Vicia faba L.) y Soya (Glycine Max L. Merr) por proceso de cocción extrusión. Tesis para optar el grado de Magister Scientiae. (Escuela de Post Grado. Especialidad de Tecnología de los Alimentos. Universidad Nacional Agraria La Molina, 2002).

[10] Dirección General de Salud Ambiental. NTS № 071. Norma sanitaria que establece los criterios microbiológicos de calidad sanitaria e inocuidad para los alimentos y bebidas de consumo humano. (Ministerio de Salud, 2008).

[11] R. Carpenter, D. Lyon, T. Hasdell, Análisis sensorial en el desarrollo y control de la calidad de productos. 2da ed. (Zaragoza, España: Acribia, S.A; 2002), pp. 121-126.

E-mail: ronnygm1@hotmail.com ylcairo@yahoo.es 